\title{
Assumption/Commitment Algorithmic Approach for Ontology Alignment
}

\author{
Said Rabah Azzam, Shikun Zhou \\ School of Engineering, University of Portsmouth, United Kingdom
}

\begin{abstract}
Ontology is the way to provide interoperability of different classes over semantic web. The better the ontology is constructed, the more are the chances for tight integration between different processes, no matter they are within the organization or are indirectly connected to organization, including external stakeholders i.e. Customers, Suppliers and Outsource services. For a properly design Ontology, it is always a mandatory step to apply different methods for Ontology Alignment. This propagates the reusability approach of a single object, thus at different instances, same property or attribute can be declared, rather going for heterogeneous environment. We have different traditional methods to align the ontology, however we are going to propose new method, adopted from the other domain, to better align the ontology. We will advance the concept by introducing algorithm which can help implementing the solution.
\end{abstract}

Keywords: Business Functions; Business Model in Semantic; Business Processes; Enterprise Architecture; Interconnected Services in Semantic; IT Alignment; OWL Business Model; OWL-S; Organization Structure; Ontologies; Service Oriented Architecture;

\section{Introduction}

To step in the business world, the entrepreneurs may have good idea to induce in the market. The idea may penetrate well, and that the customer size may increase rapidly. However the issue lies that what if the customers' size increases rapidly, what if we get a competitor's strong response, how internally the companies are built to challenge accordingly, what external factors can impact strongly to let down the vision and mission, and how to continue providing prompt services to end-users.

With this paper, the idea is to introduce the concept of what hierarchy should an organization follow, to directly cope-up with them according to the best practice, so not much time in thinking or evaluation of different practices are put into. Publishing the same in the open-source environment in the form of Semantic Web by following OWL Ontology will let it be modified by different stakeholders around the world to optimize the structure further, in order to come up with streamlined organization hierarchies around the world. The same may also help in introducing ISO standard to better rate the efficiency of the organization

\section{Previous Work}

Business Processes Modeling itself is a problematic task, and applying semantics to it is much more difficult. As rightly mentioned in [5] , adding semantics to business process, though a tough task, but to make it machine understandable, it is the only way out. Also, issue pertaining documenting the business processes, and their purpose is well define in [6], which itself is challenging. Work over organizational ontology was done previously in [7], where business terms are well defined. Other than that, a relationship is shown among different integration components in [8]. An environment to model is proposed [9], while multi-level is followed from [10].

With heterogeneous knowledge representation and reasoning with agent based services, provided in conducted research [11], we have a method of defining agents that can be connected to systems to develop organization structure in the form of machine readable format. But before agents, well defined process [12], are extremely important. Familiar semantics of agents for business processes [13] are mandatory for execution-based process ontology. However, manufacturing industry is comprehensive in relation to other industries, which is complex to get into Ontology [14]. Thus, crossfunctional integration, well provided in [15], is a must for such organizations, being inter-related with other industries. But agents need communication protocol to have built relationship with Organization [16], before providing semantic annotation.

After agents, business components are integral part of making the entire structure to machine readable. Information systems are being made to make business components reusable element [17]. Therefore, because of reusability, the semantic notions can be applied. The concept is repeatedly endorsed in [18], [19], and [20].

Changes within the processes may lead to changes in structure of an organization as well. Thus, accordingly organization restructure becomes mandatory. If semantics are well defined, it will 
automatically be done. BPM approach is therefore required for effective implementation. OWL-S is therefore recommended for defining enterprise levels semantics. However, for short term incorporation of changes, methodology can be referred. Changes however, make it cumbersome to incorporate within structure, being discussed numerously, explained well of how should be changed efficiently in distributed environment.

Though, it is rightly defined that a process should be followed to make a structure. That is, going with Business Model, accordingly working over the application and then checking if financial in favor to organization. However, it should better be in a position if organization is rightly defined, and then down the line, linking other components. IT Capabilities, is a mandatory for comprehensive architecture. Also, external influences plays major role in getting the hierarchy well define, semantically.

Regarding proposing the new method, a related work has already been done. Rely/Guarantee was utilized in distributive computing area, where shared variable is utilizing in concurrent programs [1]. The proposed logic can be automated easily [2]. In it, a process is divided into sub-systems [3] so as to classify outcome of each process. Shared variable in parallel processing is the most common method for composed systems to work efficiently [4] [5].

With Rely/Guarantee, assumptions and constrains can have constraints on single step that includes if which one of the two parallel processes would be executed. However, with the advent of assumption/commitment [6] and its refined version later [7]. This says that the outcome of one of the process is actually the input of the other process, provided that both the processes should satisfy the environmental factor.

\section{Ontology}

To create interoperability in the semantic world of all the functions/classes over the web, people around the globe worked on different technologies. In the same line, the recent research being done is on OWL Ontology, which is refined to DL and then to elaborative way, to actually develop the semantic web. Through this approach, one designs the process or process steps or entire process to perform specific function. For example, online transaction through VISA service is developed over Ontology. Thus, no matter which bank is providing the feature, the same can be replicated. Thus, a semantic web is well published with the scenario. Further, if we go for having semantics for each image uploaded over the web, if there exist the Ontology, we can easily go searching for an image, which currently is not being done, and is a challenging task.

\section{Ontology Alignment}

Alignment refers to coming up with such an Ontology that helps in proper interoperability of the classes. With the methods, we try to remove the duplication, and thus replicate the same class which is having same semantics. That will help for example, searching for particular semantics and unique search result can come up. The whole idea of effective Ontology therefore derives from the Ontology Alignment

\subsection{Terminology Technique}

This technique caters the alignment through the terminology or syntax of the class. The entire ontology is traversed, and each class is checked for its recurrence. The runtime is therefore $\mathrm{O}(\mathrm{n} 2)$. It's a time consuming and might not align the ontology, as not all syntax can simply be mapped.

\subsection{Structural Technique}

This technique deals in matching structure of each class in Ontology. This means that attributes of each class are matched, that may result in the similarity of one or more than one attributes to be matched. Thus finding similarities and coming up with the ideal similar class generalizes the class. At the end, it results in aligned ontology.

\subsection{Extensional Technique}

This technique deals in aligning the classes in ontologies, depending on their instances. So, the similar instances can be compared and if found with the same value, their classes are made similar. This results in aligned ontology, which is better than the structural technique.

\subsection{Semantic Technique}

It can further be elaborated in a way that the first stage should be testing the class on syntax basis and then comes to the local semantics of particular class. If it doesn't match, try to find the class in global semantics. This will apply interoperability on the defined classes. For being effective, reference of syntax and its semantics should be followed by same repository. Thus, proper Descriptive Language is mandatory for such technique

\section{Tools for Ontology Alignment}

\subsection{Utilization}

We have available different tools available in the market that can create OWL Ontology of the specific 
scenario/domain to create interoperability in semantic web. There are different add-ons that can help in aligning the ontology, but all in traditional way. We checked different websites that publishes add-ons for Protégé as well. However we have unable to find any of the algorithms which is similar to ass/com method. How it can be there if we are proposing this new method i.e. introducing an idea of Parallel Computing in Ontology Alignment. However, if we go implementing the same it will be a bit difficult to design and implement the same. As computer based solution runs on the systems that are perfectly provided in the frame of which the computer program understands, it is always difficult to architect data in a way to develop ontology, the basis of which will be the computer program to let it align.

We researched the web as well as found different applications that actually proposes the Ontology Alignment, but all by through the traditional methods. Although there should be a direct availability of such feature within the Main tabs of the software e.g. Protégé. This is because, alignment is the main part of any ontology, while if the ontology is not aligned, there are high chances of duplication of the data types. Thus each data can be associated with different data type and thus can have different semantics while it will not be functional as interoperable for globally published classes.

\subsection{Reason to Incorporate}

Although, it is difficult to design the implementation of Ontology Alignment to actually align the ontology as per the Ass/Com method, it is also difficult to design the Ontology according to the architecture that our implementation will understand.

However, as other traditional method which includes Semantic method, Structural Method, Terminological Method, and Expansion Method, are provided as an add-on to the Protégé and other OWL Ontology development toolkits, and Ass/Com is a way better way to align the ontology, which make almost perfectly interoperable classes over semantic web for a better exchange of information, we consider it to be the MUST TO HAVE tool for the OWL Ontology developer to let it align on the fly.

The other advantage will be that it can easily interconnect with other Business Process Management (BPM) tools, to let it known the inputs and outputs, while integrated systems, actors and position types can better be analyzed and integrate for effective communication.

We therefore recommend to have ass/com method as a mandatory tool for Ontology Alignment within the standard system to enable OWL Ontology developers to align their Ontology on the fly, and publish it so that the others can utilize the same, while information can be published over semantic web, making each attribute unique for better searching capabilities and information interoperability.

\section{Unaligned Classes}

Such classes that remain same after being applied the different techniques of Ontology Alignment are classified as Unaligned Classes. Although that is not mandatory to get each and every class of Ontology is aligned, however, we have observed that many of the classes can be aligned. However, such classes are aligned because of the ineffective methods being applied to that Ontology. If we are intended to publish the Ontology to create a semantic web, we must have a replica or semantically same class already published. Thus, classes that remain unaligned after the application of different approaches to align the Ontology are classified as Unaligned Classes.

\section{Rely/Guarantee Approach}

\subsection{Introduction}

In the past, it was easy to make an output of a system as an input of another system. This is because all the programs being generated were actually based on sequential methods. That is, the systems always rely on the output of its dependent system. However, when it comes to parallel processing, more research was performed which results in providing solution as an integral way, which was previously being done independently.

Rely-Guarantee, or in other words Assumption/Commitment is a method which assumes that not only a component is verified by just satisfying all of its commitments, but also verifies all the assumptions, being exposed from the internal or external environment.

This can better be explained with the real-life example that if we want to have dinner, we can either go out to some restaurant, can cook at home, or can place order to get it delivered at the door-step. Now, the process of having dinner is dependent on either or the commitments from the mentioned processes. For first option, we need to drive way to the restaurant to reach the destination, so "Traveling" is the process which needs to be committed. Similarly, if we want to cook at home, we first need to go for "Shopping" to buy ingredients. The process commitment will enable us to cook or Initiate cooking process, so that dinner system can be practiced. The last option is to let the dish be delivered at home. For that, two commitments, a phone call and book and order while another process by the delivery-boy to bring it to your doorstep, should be committed so have dinner. 
So, if all the assumptions from the environment are verified, while the commitments are fulfilled, the system can have its input to ignite the system. In other words, all pre-requisite systems should have commitments in order to provide feed to dependent system to let the process flow

\subsection{Rule of Thumb}

So, rely guarantee works on the rules that if the first state satisfies the initiating process and all the state being changed are relying (R), then every final state will satisfy the final process and every next state within the processing is guaranteed $(\mathrm{G})$

$$
\mathrm{R}, \mathrm{G} \vdash\{\mathrm{P}\} \mathrm{C}\{\mathrm{Q}\} \ldots \text { (1) }
$$

\subsection{Architecting Rely/Guarantee}

Any analyst can better come up with the systems and apply the assumption/guarantee method to align the ontology of that particular system. For that, the analyst first have to come up with the no. of systems exists within entire system. This means to, bifurcate the entire process into small systems which are dependent on each other, if related. The focus while deriving the sub-systems should be on how the system verifies the initial process, and all subsystems do rely on prior system to come-up with the consequence.

The technique can be adopted to align the ontologies. That creates the possibility of aligning such ontologies that cannot be aligned with the traditional methods. We will further discuss different methods to align the ontologies in different ways. Further, with the methods, we can also automate the alignment process by developing a program that can align ontology accordingly

\subsection{Parallel Rule}

With parallel rule method, we mean to have different processes being run at the same time, i.e. simultaneously. We can better explain it with the following example.

Suppose we have to come up with the minimum no. within a provided list of nos., say 100 . If we go with the sequential processing to identify minimum no. though an environment for parallel execution is available. We need to traverse entire hierarchy, the complexity of which will become $\mathrm{O}(\mathrm{n})$. However, with parallel rule, we can execute matching algorithm by splitting the number range in even and odd values. Each list will have 50 nos. Then we can execute our matching algorithm on both the list in parallel which results in half of the time as was done earlier. At final, we have two values of the two lists. We can therefore conclude that the smallest no. among two identified nos. is the lowest in the range.

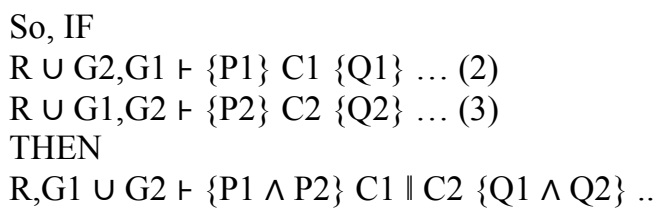

Later, we will apply the Parallel Method on one of our modeled class to get its outcome and compare it with the other proposed method for Rely/Guarantee.

\subsection{Rule of Consequences}

In rule of consequences, we assume that if the dependent state satisfies with all the environment assumptions and the next state satisfies the same assumptions, the outcome will also guarantees to satisfy the all the assumptions.

Therefore, if Initial Process (P) satisfies all the assumptions (R), while the final process (Q) satisfies all the assumptions $(\mathrm{R})$, which was earlier satisfied by $P$ as well, and if the effect is guaranteed and is contained in $(\mathrm{G})$, then

$$
\mathrm{R}, \mathrm{G} \vdash\{\mathrm{P}\} \mathrm{c}\{\mathrm{Q}\} \ldots(5)
$$

In other words, if all the processes are relying, and feeding input to other process which also satisfies the assumptions, than within the two processes, guarantee exists. The change states will be maintained in Guaranteed state $(G)$

With a real-life example, we can better explain the method. For instance, if we have a process to spend holidays (Process 0), we need to book the tickets (Process 1), need to purchase clothes (Process 2), and need to purchase fresh-food (Process 3). For Process 0, we first need to proceed for Process 1 as its outcome will inform either to trigger Process 2 and Process 3. This is the consequence-rule way of assignment/commitment. However, Process 3 and Process 2 can be run in parallel as Process 3 can be triggered by ordering, while Process 2 is what we our-self have to do. So, in a same time, both the processes will be executed, thus being followed in parallel. However, this parallel execution is a consequence of Process 1. This means that Process 1 satisfies the assumptions, while Process 2 and 3 satisfies assumptions only if Process 1 satisfies, i.e. If tickets are booked, than the shopping and food purchase is executed, while if tickets are not booked, both the dependent processes will not be executed. This endorses applicability of Rely-Guarantee.

In other words, IF

$\mathrm{R} \subseteq \mathrm{R}^{\prime} \mathrm{R}^{\prime}, \mathrm{G}^{\prime} \vdash\{\mathrm{P}\} \mathrm{C}\{\mathrm{Q}\} \mathrm{G}^{\prime} \subseteq \mathrm{G} \ldots$ (6)

THEN

$\mathrm{R}, \mathrm{G} \vdash\{\mathrm{P}\} \mathrm{C}\{\mathrm{Q}\} \ldots(7)$ 


\section{Algorithm}

The following is the algorithm to come up with the proposed way of aligning Ontology

1. Declare Aligned Classes Container O

2. Declare Checking Container A

3. Declare Checking Container B

4. Loop - For each Class X in an Ontology

a. Gather Input Parameters

b. Gather Output Parameters

c. Add collected parameters from step (4.a) and step (4.b) in Container A, declared in step (2)

d. Start Nested Loop - Traverse to Entire Ontology

i. Gather Input Parameters

ii. Gather Output Parameters

iii. Add collected parameters from step (4.d.i) and step (4.d.ii) in Container B, declared in step (2)

iv. Compare Container A and Container B

v. IF Equals

1. Add Class $\mathrm{X}$ to Container $\mathrm{O}$

vi. End IF

e. End Nester Loop

5. End Loop

6. Loop - For each Class Y in an Ontology

a. Check existence of Class $\mathrm{Y}$ in Container $\mathrm{O}$

b. IF exists

i. Replace Class $\mathrm{Y}$ with reference value from Container $\mathrm{O}$

c. END IF

7. End Loop

\section{Suggestions}

We suggest having a separate tool for Ontology that functions on the following main parts

\subsection{Availability of Deployed Attributes over Semantic Web}

By availability of deployed attributes, we mean to have a tool that can connect to semantic web server and able to access all attributes to reutilize the attribute within our class, having same semantics Availability of Deployed Classes over Semantic Web By availability of deployed classes over semantic web, we mean to have a tool that not only have access to globally published attributes but also the classes to reutilize the properties defined within the class, to instantiate object of particular class, thus having same semantic object, related to same class. Thus interoperability is achievable.

\subsection{OWL Ontology Importer}

By OWL Ontology importer, we mean to have functionality within a tool that can import already defined OWL Ontology, and after parsing each attribute and classes while importing, provide suggestions of the replaceable objects, to directly convert it into the globally published attributes/classes

\subsection{Integration with BPM Tools}

By integration with business process management (BPM) tool, we mean to have direct connectivity with the tool, to pick the connected resources, so as to identify pattern of which object is actually initiating the project, and which object is at the receiving object. Thus, with such information, we can have known semantics of the particular class or attribute

\subsection{Ass/Com Alignment}

By our proposed method, ass/com algorithm provided in the earlier section, we can align the ontology by actually analyzing the dependent objects of particular class, that what input the class has been receiving, while what output the class is providing, who is actually sending the input information and who is receiving the processed information for further processing. This helps in identifying the pattern and thus proposing the aligned ontology, as discussed above in earlier sections.

\section{Conclusion}

Extensive research was done on traditional method earlier, while it is difficult to apply on entire Ontology, which usually is complex. With the new proposed method of Rely/Guarantee, it is expected to develop grounds for further research in this area for effective alignment of the ontology. We have also covered what algorithm can best be adopted in order to implement the solution and incorporate the tool within the Ontology Alignment software. We also covered the comprehensive tool that Alignment software should have in order to make an Ontology effectively developed as well as tested before publishing over semantic web.

\section{References}

[1] A Modelling Languages and Semantic Integration in Business and IT Works. Zimmerli,

[2] An Ontology-Based Approach for Supporting Business-IT Alignment. Csaba, Jennifer, Karl. 2010. 
[3] Architecture, Business IT Alignment from Business Model to Enterprise. Fritscher, Boris. 20xx.

[4] Better Alignment of IT with Business. Jenz, Dieter E. 2003.

[5] Process Modeling. B. Curtis, M.I. Kellner and J. Over. 1992.

[6] The Enterprise Ontology. M. Uschold, M. King, S. Moralee, Y. Zorgios. s.l. : The vKnowledge Engineer Review 13(1).

[7] An Accounting Object Infrastructure For KnowledgeBased Enterprise Models. McCarthy, G. Geerts and W.E. s.1. : IEEE Intelligent Systems and Their Applications, 1999.

[8] A bpmo based semantic business process modelling environment. M. Dimitrov, A. Simov, S. Stein, and M. Konstantinov. s.1. : In Proc. of SBPM, 2007.

[9] Semantic EPC: Enhancing Process Modeling Using Ontology Languages. Fellmann, O. Thomas and M. s.l. : In Proc. of SBPM, 2007.

[10] A Semantic Framework for Business Process Modeling Based on Architecture Styles. Mohammad Ebrahim Khalij, Shaorouz Moaven, Jafar Habibi, Hamed Ahmedi. s.l. : IEEE/ACIS 11th International Conference, 2012.

[11] Research on Business Processes with Semantic Web Technologies. Qiumei Pu, Xiuqin Pan, Yue Zhao, Xiaona Xu. s.l. : Ninth International Symposium on Distributed Computing and Applications to Business, Engineering and Science, 2010.

[12] Linking Business Goals to Process. Markov, I. and Kowalkiewicz, M. s.1. : EDOC, 2008.

[13] Ontology-Based Translation of Business Process Models. Norton, B., Cabral, L. and Nitzsche, J. s.l. : ICIW, 2009.

[14] Manufacturing enterprise business process. Fanbo, Ye and Xianghai, Ding. s.l. : GSIS, 2009.

[15] An Ontology Based Method forBusiness Process Integration. Fan, Shuchuan, Zhang, Li and Sun, Zian. s.l. : IESA, 2009.

[16] Agent-Based Electronic Market With OntologyServices. Silva, N., Viamonte, M.J. and Maio, P. s.l. : ICEBE, 2009.

[17] An ontology-based method for semantic integration of Business Components. Larbi Kzaz, Hicham Elasri, Abderrahim Sekkaki. 20xx.

[18] Business Components", Business Component-Based Software Engineering. Barbier F., Atkinson C. s.l. : Kluwer, 2002, Vol. 705.

[19] A Model for Integration of Business Components. Kzaz L., Elasri H., Sekkaki A. s.l. : International Journal of
Computer Science \& Information Technology, 2010, Vol. II.

[20] Ontology Learning for the Semantic Web. Maedche A, Staab S. s.1. : IEEE Intelligent Systems, 2001. 